\title{
Temperature Dependence of Soret coefficient in Aqueous and Non-aqueous Solutions of Pullulan
}

\author{
Yuki Kishikawa, ${ }^{\dagger}$ Rio Kita, $^{* \dagger}$ and Simone Wiegand ${ }^{\S,}$ \\ Department of Physics, Tokai University, Hiratsuka, Kanagawa 259-1292, Japan \\ Forschungszentrum Jülich GmbH, IFF - Weiche Materie, D-52428 Jülich, Germany
}

\begin{abstract}
We presents experimental results of the temperature dependence of the LudwigSoret effect for pullulan solutions. The Soret coefficients of $5.0 \mathrm{gL}^{-1}$ pullulan in water and in dimethyl sulfoxide (DMSO) were determined in the experimental temperature range between $20.0^{\circ} \mathrm{C}$ and $50.0^{\circ} \mathrm{C}$ by means of thermal diffusion Forced Rayleigh scattering (TDFRS). The sign of the Soret coefficient of pullulan in water is negative at room temperature, which indicates that the pullulan molecules migrate to the warm side of the fluid. The Soret coefficient of pullulan increases steeply with increase of the solution temperature and shows a sign change from negative to positive at $41.7^{\circ} \mathrm{C}$. The positive sign of the Soret coefficient means the pullulan molecules move to the cold side. In contrast to the aqueous solution, the solution of pullulan in DMSO shows a very weak temperature dependence of the Soret coefficient and has always a positive sign. In addition to the TDFRS experiments we performed also light scattering (LS) experiments for the pullulan solutions under homogeneous temperature condition in a temperature range between $20.0^{\circ} \mathrm{C}$ and $55.0^{\circ} \mathrm{C}$. The thermodynamic properties of pullulan solutions obtained by LS show no pronounced correlation with the thermal diffusion behavior of pullulan. These results indicate the existence of a special role of interactions due to solvation on the temperature dependence of the thermal diffusion behavior of polysaccharide solutions.
\end{abstract}

\footnotetext{
${ }^{\dagger}$ Tokai University.

${ }^{\ddagger}$ Forschungszentrum Jülich GmbH.

*E-mail: rkita@keyaki.cc.u-tokai.ac.jp

${ }^{\S}$ E-mail: s.wiegand@fz-juelich.de
} 


\section{INTRODUCTION}

Ludwig-Soret effect, also known as thermal diffusion or thermophoresis in the case of colloidal particles, describes the transport of mass which is induced by a temperature gradient. ${ }^{1-3}$ For polymer solutions, under a stationary temperature gradient, the macromolecules migrates to the cold or the warm side of the fluid and form a stable concentration gradient. The magnitude of the Soret coefficient of polymers is much larger than that of the mixtures composed of small molecules. This fact provides a practical application of polymer separation, e.g., thermal field-flow fractionation (thFFF) which has been applied for characterization of polymer solutions. ${ }^{4-6}$ Although the Ludwig-Soret effect of fluid mixtures has been studied for a long time since the exploring works of Ludwig and Soret, the understanding of the molecular mechanisms of the phenomena is still lacking. For complex systems such as polymer solutions and colloidal suspensions, there is no complete theory to predict the direction of the solute molecules in a temperature gradient. ${ }^{7,8}$ Only specific aspects as the interaction contribution or charge effects in highly diluted solutions of colloidal dispersions have been considered so far. ${ }^{9-14}$

The Ludwig-Soret effect for a binary fluid mixture is described by the flux $\mathbf{J}_{\mathbf{1}}$ of component 1 contains contributions of the concentration and the temperature gradient as follows ${ }^{15}$

$$
\mathbf{J}_{1}=-\rho D \nabla w-\rho w(1-w) D_{\mathrm{T}} \nabla T .
$$

Here, $\rho$ is the density of solution, $D$ the translational mass diffusion coefficient, $D_{\mathrm{T}}$ the thermal diffusion coefficient, $w$ the mass fraction of component 1 , and $T$ the temperature. In a steady state where the mass flow vanishes $\left(\mathbf{J}_{\mathbf{1}}=0\right)$, the concentration gradient is given by

$$
\nabla w=-S_{\mathrm{T}} w(1-w) \nabla T
$$

where $S_{\mathrm{T}}=D_{\mathrm{T}} / D$ is the Soret coefficient. The sign of the Soret coefficient indicates the direction of the flux of component 1 . The positive sign of $S_{\mathrm{T}}$ means that the component 1 migrates to the cold side. In general for organic polymer solutions, the polymers due to their heavier mass and larger size compared to the solvent molecules move to the cold side. ${ }^{16-19}$

Some aqueous solutions of polymers show negative Soret coefficients, which correspond to a migration of the polymers to the warm side. ${ }^{20-25}$ It has been revealed that a sign of $S_{\mathrm{T}}$ of the polymer in solution can occur as function of the solution temperature as well as of the solvent composition. ${ }^{26-30}$ For liquid mixtures composed of small molecules thermally induced sign 
change of $S_{\mathrm{T}}$ have been studied by several researchers ${ }^{31-34}$, sometime those sign changes could be correlated with structural changes in the liquid mixtures. For polymer solutions and colloidal suspensions sometimes sign changes occur und bad solvent conditions. ${ }^{20,27}$. Recently Stadelmaier and Köhler performed a systematic experimental investigation of the thermal diffusion in diluted polystyrene solutions.? They scrutinized the crossover from small molecules to high molecular weight polymers in seven different solvents and found for two solvents a sign change with chain length.

The temperature dependence of the Soret coefficient and thermal diffusion coefficient for polymer solution is system dependent. Theoretically it has been predicted that the temperature dependence of the thermal diffusion coefficient is proportional to $1 / T^{2}{ }^{35-37}$, however the experimental data show a more complicated temperature dependence. For poly(styrene) [PS] solutions, the temperature dependence of $S_{\mathrm{T}}$ has been studied by Brimhall et. al. ${ }^{38}$ with thFFF experiment. They showed that the thermal diffusion coefficient is proportional to the temperature. Mes et al. discussed the temperature dependence of of thermal diffusion coefficient of PS solutions taking into account the thermodynamic interaction parameter $\chi$ of Flory-Huggins equation. ${ }^{39}$ They found a slight increase of the Soret coefficient as a function of temperature. Under poor solvent conditions close to a critical temperature the Soret coefficient diverges due to the critical slowing down, but $D_{\mathrm{T}}$ remains constant. ${ }^{40-42}$ For aqueous solutions of polymers the Soret coefficient shows often a complicated temperature and concentration dependence. The Soret coefficient $S_{\mathrm{T}}$ of poly(ethylene oxide) $[\mathrm{PEO}]$ in the solvent mixture ethanol/water increases with temperature, whereas the slope is positive and decreases with increasing water content. ${ }^{21,22,26,27}$ The Soret coefficient $S_{\mathrm{T}}$ changes sign from negative to positive with increasing temperature for ethanol/water mixtures with a high ethanol content, while $S_{\mathrm{T}}$ is always positive for PEO in pure water. A similar temperature dependence of $S_{\mathrm{T}}$ has been observed for dextran in water. It shows a negative value at room temperature while it is positive at higher temperatures. The addition of urea to the aqueous solution of dextran increases $S_{\mathrm{T}}$ and sign changes occurs at lower temperatures. The reason is probably that the addition of urea destroys the hydrogen bonding network. ${ }^{24}$ Thermally induced sign changes of the Soret coefficient can also be found in biological polymers, proteins, DNA and polysaccharide. Piazza and coworkers found the sign change of lysozyme solutions ${ }^{23}$ and Braun and coworkers revealed the sign change aqueous solutions of DNA. ${ }^{29}$

Poly $(N$-isopropylacrylamide) [PNiPAM] is one of the water soluble polymers, which has been studied systematically in water and in mono-alcohols. ${ }^{25,28,43}$ PNiPAM in water has a $\Theta$-temperature 
of $30.6{ }^{\circ} \mathrm{C}$ where the second virial coefficient is zero. At the lower temperatures water is a good solvent, while it becomes a poor solvent above the $\Theta$-temperature. The temperature dependence of $S_{\mathrm{T}}$ shows a strong enhancement at the $\Theta$-temperature but the sign of $S_{\mathrm{T}}$ is always positive. ${ }^{43}$ While for PNiPAM in ethanol solution, $S_{\mathrm{T}}$ of PNiPAM decreases with temperature and changes the sign at $34{ }^{\circ} \mathrm{C}$ from positive to negative. ${ }^{28}$ Here, it should be noted that PNiPAM in ethanol is so far the only system, with a negative slope for the temperature dependence of $S_{\mathrm{T}}$. All aqueous solutions of PEO, dextran, lysozyme, and DNA show a positive slope. This behavior indicates that the interactions among the polymer molecules and solvents have a key role for thermal diffusion.

Recently it has been shown that biopolymers, DNA, protein and polysaccharide show a sign change of the Soret coefficient as function of temperature. Duhr et al. studied DNA in solution and found a sign change of $S_{\mathrm{T}}$ around $2{ }^{\circ} \mathrm{C} .{ }^{29}$ Iacopini et al. reported the Soret effect for lysozyme solutions which show a sign change of $S_{\mathrm{T}}$ from negative to positive with increasing temperature. ${ }^{23,44}$ Sugaya et. al. reported a sign change for dextran in water. ${ }^{24}$ It is interesting to study the relationships between physiological functions and thermal diffusion for biological polymers, because the biopolymers play their functions under complex conditions subjected to various fields. However the number of studies of the Ludwig-Soret effect for biological polymer are limited. In this study, we will report the experimental study of the Ludwig-Soret effect for solutions of pullulan, which is one of the standard samples of polysaccharide composed of $\alpha$-D-(1 $\rightarrow 6)$-linked maltotriose. In order to study the contribution of the hydrogen bonding on Ludwig-Soret effect, water and dimethyl sulfoxide [DMSO] were used as solvents. DMSO is a polar aprotic solvent which does not form hydrogen bonds. Pullulan and dextran are composed of glucose as basic constituent, therefore it might give insight into the chemical contribution to the thermal diffusion. Additionally, we studied the solution properties of pullulan under homogeneous temperature condition by light scattering (LS) which yields fundamental properties such as the mass and size of the polymers and the thermodynamic parameters of the solutions.

\section{EXPERIMENTAL SECTION}

Materials. Pullulan (Hayashibara Co., PF20) was purified three times by a methanol precipitation from the aqueous solutions. The molar mass of pullulan was measured by static light scattering and we obtained $M_{\mathrm{w}}=440 \mathrm{~kg} / \mathrm{mol}$. Distilled and deionized water was prepared by a Milli-Q system. DMSO of an analytical grade was used as solvent. For the Thermal diffusion 
forced Rayleigh scattering (TDFRS) measurements $5.0 \mathrm{~g} / \mathrm{L}$ pullulan in water and in DMSO were prepared. To accieve a sufficient absorption of the laser light we added a small amount of the dye, Basantol Yellow (BASF) or quinizarin(Sigma-Aldrich).

\section{Methods.}

The experimental setup of TDFRS has been described in detail elsewhere. ${ }^{45,46}$ (???) In brief,the optical grating is written by the interference of two beams of theargon ion laser $(\lambda=488 \mathrm{~nm})$. The interference grating is rea out by a He-Ne laser $(\lambda=632.8 \mathrm{~nm})$. The intensity of the difracted beam was measured by a photomultiplier. A mirror mounted on a piezocrystal was used for phase shifting and stabilzation to obtain the heterodyne signal. The TDFRS measurements were crried out in the temperature range from 20 to $50^{\circ} \mathrm{C}$. The temperture of the sample cell was controlled by circulating water from athermostat bath with an uncertainty of $0.01{ }^{\circ} \mathrm{C}$. The sample solutions were filtered directly into the optical quartz cell with $0.2 \mathrm{~mm}$ path length through $0.22 \mu \mathrm{m}$ membrane filter.

Light scattering (LS) experiments were carried out in an angular range of $25^{\circ}<\theta<150^{\circ}$. A He-Ne laser with a wavelength of $\lambda=632.8 \mathrm{~nm}$ was used as light source. The intensity correlation function $g^{(2)}(t)$ was destected with a ALV-6000E correlator. The measurements were carried out in a temperature range from 20 to $55^{\circ} \mathrm{C}$. The temperature of the sample cell was controlled by a circulating water bath with an uncertainty of $0.02^{\circ} \mathrm{C}$. All samples were kept at the measurement temperature for at least one hour to ensure thermal equilibrium before starting the data acquisition.

The refractive index increments with respect to the mass fraction $(\partial n / \partial w)$ and to the temperature $(\partial n / \partial T)$ need to be determined for evaluation of $S_{\mathrm{T}}$ and $D_{\mathrm{T}}$ in the TDFRS experiment as described in Eq. 3. Here, $w$ indicates the mass fraction of the polymer in solvents. The value of $(\partial n / \partial c)$ is also required for the analysis of the static LS data. The refractive index increments $(\partial n / \partial T),(\partial n / \partial w)$ and $(\partial n / \partial c)$ of pullulan in water and in DMSO were measured with a scanning Michelson interferometer operating at a wavelength of $632.8 \mathrm{~nm} \cdot{ }^{47}$ All experiments for the determination of the increments were carried out at the room temperature. The value of $(\partial n / \partial T)$ for pullulan solutions with $w=5.0 \mathrm{~g} \mathrm{~L}^{-1}$ was obtained from interference signals in the temperature range of $\pm 0.5^{\circ} \mathrm{C}$ around the desired temperature Is this correct $\pm 0.5^{\circ} \mathrm{C}$ or is it $\pm 0.25^{\circ} \mathrm{C}$ ???. The temperature was controlled by circulating water bath with an uncertainty of $0.02^{\circ} \mathrm{C}$. 


\section{WORKING EQUATION}

The normalized heterodyne signal intensity of TDFRS experiments, $\zeta_{\text {het }}(t)$ is related to the Soret coefficient $S_{\mathrm{T}}$ and translational diffusion coefficient $D$ as follows:

$$
\zeta_{\text {het }}(t)=1+\left(\frac{\partial n}{\partial T}\right)^{-1}\left(\frac{\partial n}{\partial w}\right) S_{\mathrm{T}} w(1-w)\left[1-\exp \left(-q^{2} D t\right)\right]
$$

Here, $t$ is the time, $n$ the index of refraction, $q$ the scattering vector.

For dilute polymer solutions the Rayleigh ratio $R(\theta)$ of LS experiments is expressed by

$$
\frac{K c}{R(\theta)}=\left(\frac{1}{M_{\mathrm{w}}}+2 A_{2} c\right)\left(1+\frac{1}{3} R_{\mathrm{g}} q^{2}\right)
$$

where $K$ is the optical constant $\left[K=4 \pi^{2} n^{2}(d n / d c)^{2} / N_{\mathrm{A}} \lambda^{4}\right], A_{2}$ the second virial coefficient and $c$ the concentration of the polymer. The autocorrelation function of the scattered light $g^{(2)}(t)$ is related to the electric field autocorrelation function $g^{(1)}(t)$ by the Siegert relation

$$
g^{(2)}(t)=B\left[1+\beta\left|g^{(1)}(t)\right|^{2}\right]
$$

where $B$ and $\beta$ are the baseline and a machine constant, respectively. In general, $g^{(1)}(t)$ is expressed with the distribution function $\mathrm{G}(\Gamma)$ of the decay rate $\Gamma$ as

$$
g^{(1)}(t)=\int G(\Gamma) \exp (-\Gamma t) d \Gamma .
$$

Here, $\int \mathrm{G}(\Gamma) \mathrm{d} \Gamma=1$. That is, $g^{(1)}(t)$ is the Laplace transform of $\mathrm{G}(\Gamma)$. The cummulant expansion is used to obtain average decay rate $\bar{\Gamma}$,

$$
\ln \left|g^{(1)}(t)\right|=-\bar{\Gamma} t+\frac{1}{2 !} \mu_{2} t^{2}-\frac{1}{3 !} \mu_{3} t^{3}+\cdots
$$

where $\bar{\Gamma}=\int \Gamma G(\Gamma) \mathrm{d} \Gamma$ and $\mu_{i}$ is the $i$-th cummulant defined as $\mu_{i}=\int(\Gamma-\bar{\Gamma})^{i} \mathrm{G}(\Gamma) d \Gamma$. The normalized normalized variance is expressed as $\mu_{2} / \bar{\Gamma}^{2}=\int\left[(\Gamma-\bar{\Gamma})^{2} / \bar{\Gamma}^{2}\right] G(\Gamma) d \Gamma$. The average decay rate has the relation

$$
\bar{\Gamma} / q^{2}=D_{0}\left(1+k_{\mathrm{d}} c+\cdots\right) .
$$

Here $D_{0}$ is the translational diffusion coefficient at infinite dilution and $k_{\mathrm{d}}$ is the second hydrodynamic virial coefficient. The hydrodynamic radius $R_{\mathrm{h}}$ of the polymer is related to $D_{0}$ by the Stokes-Einstein equation, 


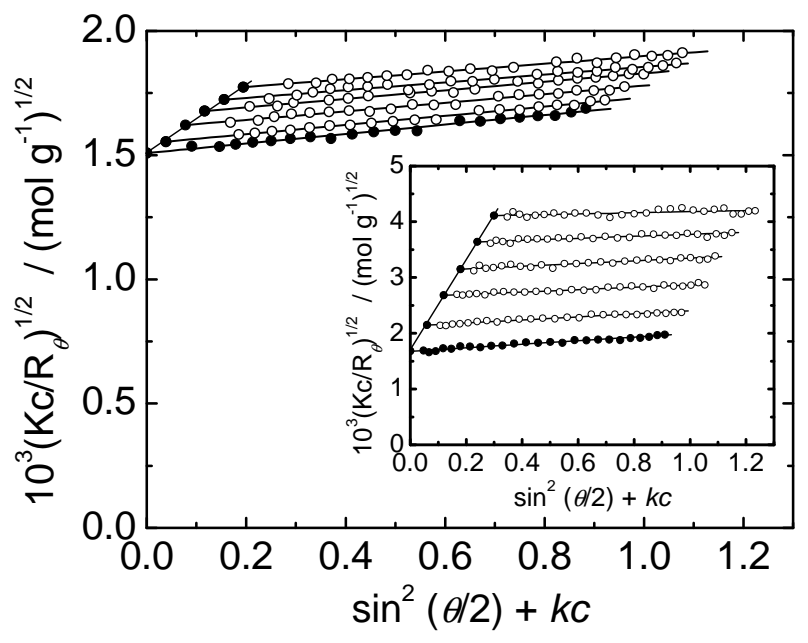

FIG. 1: Typical results of LS experiments. Berry plot for pullulan in water (a) and pullulan in DMSO (b) measured at $25.0^{\circ} \mathrm{C}$.

$$
R_{\mathrm{h}}=k_{\mathrm{B}} T /\left(6 \pi \eta D_{0}\right),
$$

where $k_{\mathrm{B}}$ is the Boltzmann's constant and $\eta$ the solvent viscosity.

\section{RESULTS AND DISCUSSION}

\section{Laser Light Scattering.}

In order to characterize the solution properties of pullulan in water and in DMSO, we performed static and dynamic LS measurements in a temperature range of $20<T_{\mathrm{e}} /{ }^{\circ} \mathrm{C}<55$ (pullulan in water) and $20<T_{\mathrm{e}} /{ }^{\circ} \mathrm{C}<40$ (pullulan in DMSO). The refractive index increments with respect to the concentration for pullulan in water and in DMSO were obtained as $(d n / d c)=0.131 \pm 0.004$ and $(d n / d c)=0.059 \pm 0.001 \mathrm{ml} \mathrm{g}^{-1}$, [***These are temporal values. ***WE ARE DOING EXPERIMENTS FOR CHECKING THE VALUES], respectively. Here, the uncertainty denotes one standard deviation. Figure 1 shows a typical result of a square plot (Berry plot) for the pullulan in water and in DMSOgive a reference here for the berry plot. The filled circles represent the extrapolated values to the zero concentrations and to the zero scattering angles. Molecular parameters were obtained by a least squares-fit to Eq. 4 and are listed in Table I. Figure 2 shows the typical results of a dynamis LS (DLS) experiment for pullulan in water and in DMSO. The distribution functions $G(\tau)$ of relaxation time $\tau$ is obtained by the CONTIN methodgive a reference here for CONTIN. The results indicate that the pullulan has a monoexponential mass distribution with 


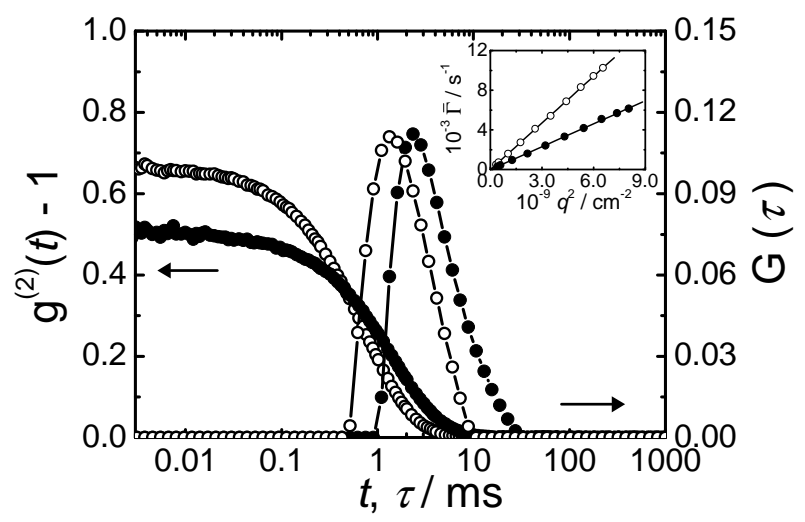

FIG. 2: Typical correlation function of scattered light $g^{(2)}(t)$ and the distribution function $G(\tau)$ obtained by dynamic light scattering for $5.0 \mathrm{~g} / \mathrm{L}$ pullulan in water (o) and in DMSO $(\bullet)$ at $T_{\mathrm{e}}=25.0^{\circ} \mathrm{C}$ and $\theta=30^{\circ}$. The inset shows the average decay rate $\bar{\Gamma}$ as a function of scattering vector $q^{2}$.

polydispersity index of $\mu_{2} / \bar{\Gamma}^{2} \approx 0.3$ as shown in Table II. The inset of Figure 2 shows the average decay rate $\bar{\Gamma}$ as a function of the scattering vector $q^{2}$. The obtained parameters such as translational diffusion coefficient $D_{0}$, hydrodynamic radius $R_{\mathrm{h}}$, and others in Eqs. 8 and 9 are shown in Table II.

The LS experiments revealed that the molecular parameters of pullulan in water and DMSO show no significant temperature dependence. In the investigated temperature range the averaged expansion factor $R_{g} / R_{h}$ of the polymer chain is $R_{g} / R_{h}=1.7$ and 1.6 for water and DMSO solutions, respectivelyWhat is the accuracy?. This result is typical for flexible coils in a good solvent with a broad molecular weight distribution. Our results are also in good agreement with previous reports. ${ }^{48,49}$ In the investigated temperature range the averaged second virial coefficient is $A_{2}=$ $1.6 \times 10^{-4} \mathrm{~cm}^{3} \mathrm{~mol} \mathrm{~g}{ }^{-2}$ for pullulan in water and $A_{2}=4.1 \times 10^{-4} \mathrm{~cm}^{3} \mathrm{~mol} \mathrm{~g}^{-2}$ for pullulan in DMSO. Therefore, both solvent can be regarded as good solvents. The chain dimensions and the interactions are constant in the investigated temperature range.

With the DLS measurements we determined the translational diffusion coefficient $D_{0}$ as function of the temperature. As shown in Fig. 3 the logarithm of $D_{0}$ decreases linearly as function of the inverse temperature. The magnitude of the diffusion coefficient of pullulan in water is larger than that of pullulan in DMSO due to the difference in the solvent viscosity $\left(\eta_{\text {water }}=0.89\right.$ and $\eta_{\text {DMSO }}=$ $2.00 \mathrm{mPa} \cdot \mathrm{s}$ at $25^{\circ} \mathrm{C}$ ). The slope is the same for both solutions and the temperature dependenc can be described by a Arrhenius type equation as

$$
D_{0}=D_{0, \mathrm{c}} \exp \left(-\frac{E_{\mathrm{D}}}{R T}\right)
$$




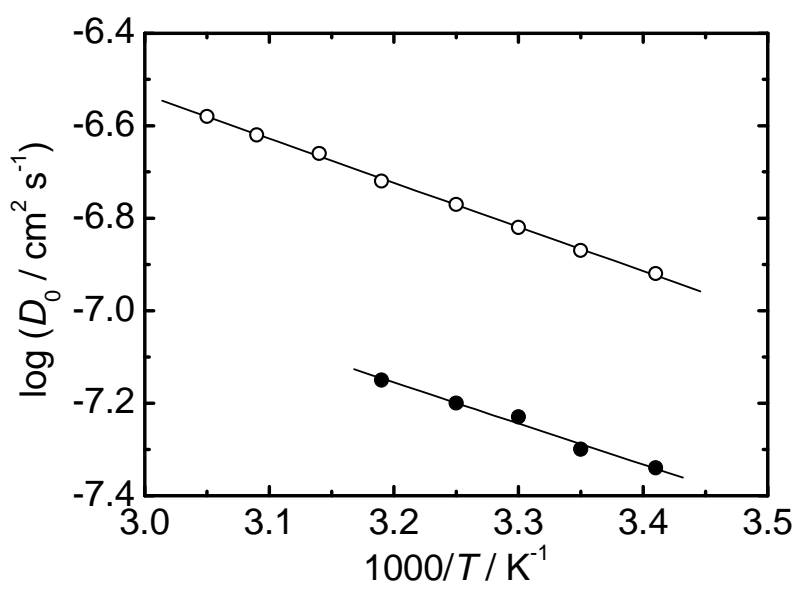

FIG. 3: Plots of $\log \left\langle D_{0}\right\rangle$ as a function of the inverse of the temperature, $1 / T$ for $5.0 \mathrm{gL}^{-1}$ pullulan in water (०) and $5.0 \mathrm{gL}^{-1}$ pullulan in DMSO $(\bullet)$ observed by DLS experiment.

where $D_{0, \mathrm{c}}$ is a constant, $R$ the gas constant, and $E_{\mathrm{D}}$ the activation energy for diffusion. The activation energy $E_{\mathrm{D}}$ was obtained by a least squares-fit to Eq. 10 as $E_{\mathrm{D}}=18.1 \pm 0.4 \mathrm{~kJ} \mathrm{~mol}^{-1}$ for pullulan in water and $E_{\mathrm{D}}=16.6 \pm 1.3 \mathrm{~kJ} \mathrm{~mol}^{-1}$ for pullulan in DMSO. The uncertainty corresponds to one standard deviation. The activation energy shows no significant difference for both solutions. The observed temperature dependence is in the diluted concentration range and is in good agreement with previous studies. ${ }^{49,50}$

\section{Thermal diffusion of pullulan.}

The normalized heterodyne signals $\zeta_{\text {het }}$ of the TDFRS experiment of $5.0 \mathrm{~g} \mathrm{~L}^{-1}$ pullulan in water and in DMSO as function of the temperature are shown in Fig. 4. Open and filled symbols refer to solutions of pullulan in water and in DMSO, respectively, and different shapes of the symbols designate different temperatures. The rapid increase of the normalized heterodyne signal $\zeta_{\text {het }}(t)$ corresponds to the establishment of the temperature gradient in a time scale of microseconds after the intensity grating has been switched at time $t=0$. At later times, the slower increasing or decreasing part of the signal indicates the establishment of the concentration gradient within a time scale of seconds. For pullulan in water, the signal curvature changes its direction from downward to upward with increasing temperature. The turnaround of the signal corresponds to the sign change of the Soret and the thermal diffusion coefficient. Whereas the signals $\zeta_{\text {het }}(t)$ of pullulan in DMSO show an increase and the curves for all temperatures overlap. This indicates that the there is no significant temperature dependence for the formation of a concentration gradient.

Figure 5 shows the temperature dependences of the Soret coefficient $S_{\mathrm{T}}$, translational diffusion 


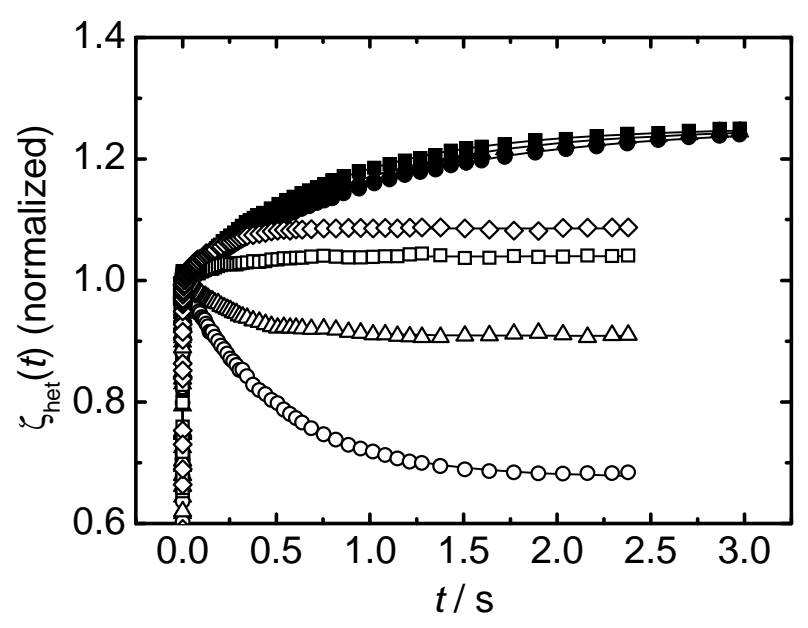

FIG. 4: Normalized heterodyne signals $\zeta_{\text {het }}$ of TDFRS experiment for $5.0 \mathrm{~g} / \mathrm{L}$ pullulan in water (filled symbols) and in DMSO (open symbols) at different temperatures. The temperatures are $25.0^{\circ} \mathrm{C}(\circ, \bullet), 35.0$ ${ }^{\circ} \mathrm{C}(\triangle, \boldsymbol{\Delta}), 45.0{ }^{\circ} \mathrm{C}(\square, \boldsymbol{\square})$ and $50.0{ }^{\circ} \mathrm{C}(\diamond)$. The solid lines on the part of concentration signal refer to the fitting function according to Eq. 3.

coefficient $D$, and thermal diffusion coefficient $D_{\mathrm{T}}$ of $5.0 \mathrm{~g} / \mathrm{L}$ pullulan in water and in DMSO. Here, $S_{\mathrm{T}}$ and $D$ were obtained by a nonlinear least-square fit of the concentration part of $\zeta_{\text {het }}(t)$ signal to Eq. 3 with the contrast factors, $(\partial n / \partial T)$ and $(\partial n / \partial w)$. The contrast factors of pullulan solutions were obtained by a Michelson interferometer as

$$
\begin{aligned}
(\partial n / \partial T) / \mathrm{K}^{-1} & =9.5759 \times 10^{-3}-8.2729 \times 10^{-5} T+2.3863 \times 10^{-7} T^{2} \\
& -2.3491 \times 10^{-10} T^{3}
\end{aligned}
$$

for $5.0 \mathrm{gL}^{-1}$ pullulan in water $\left(20<T_{\mathrm{e}} /{ }^{\circ} \mathrm{C}<50\right)$ and

$$
\begin{aligned}
(\partial n / \partial T) / \mathrm{K}^{-1} & =-8.1970 \times 10^{-3}+7.3633 \times 10^{-5} T-2.3300 \times 10^{-7} T^{2} \\
& +2.4579 \times 10^{-10} T^{3}
\end{aligned}
$$

for $5.0 \mathrm{gL}^{-1}$ pullulan in DMSO $\left(20<T_{\mathrm{e}} /{ }^{\circ} \mathrm{C}<40\right)$. The refractive index increments with respect to the mass fraction were obtained as $(d n / d w)=0.131 \pm 0.002$ for pullulan in water and $(d n / d w)=$ $0.056 \pm 0.000 \mathrm{ml} \mathrm{g}^{-1}$ for pullulan in DMSO [***These are temporal values. ***WE ARE DOING EXPERIMENTS FOR CHECKING THE VALUES] measured at $25.0{ }^{\circ} \mathrm{C}$. The thermal diffusion coefficient $D_{\mathrm{T}}$ was calculated using the relation $D_{\mathrm{T}}=S_{\mathrm{T}} \cdot D$.

At low temperatures we observe negative Soret coefficients for pullulan in water. The negative sign of $S_{\mathrm{T}}$ implys that the pullulan molecules move to the warm side. The Soret coefficient $S_{\mathrm{T}}$ 


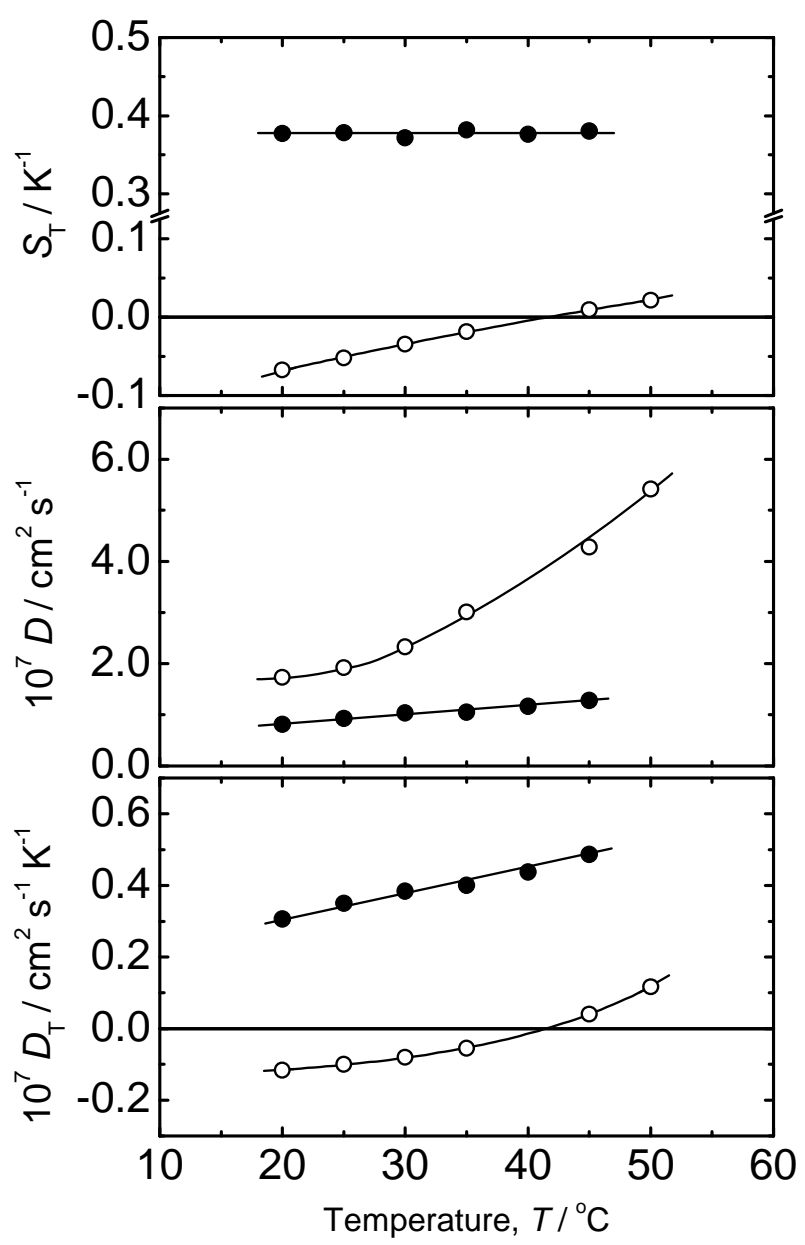

FIG. 5: Temperature dependence of Soret coefficient $S_{\mathrm{T}}$, translational diffusion coefficient $D$, and thermal diffusion coefficient $D_{\mathrm{T}}$ of $5.0 \mathrm{~g} / \mathrm{L}$ pullulan in water (०) and in DMSO (•) obtained by TDFRS.

increases with increasing temperature and the thermally induced sign change occurs at $41.7{ }^{\circ} \mathrm{C}$. Contrary, the solution of pullulan in DMSO shows a positive sign of $S_{\mathrm{T}}$ for all temperatures and no significant temperature dependence. The translation diffusion coefficient $D$ and the thermal diffusion coefficients $D_{\mathrm{T}}$ of pullulan increase with increasing temperature for aqueous and DMSO solutions. The diffusion coefficients $D$ of $5.0 \mathrm{~g} / \mathrm{L}$ pullulan at $25.0^{\circ} \mathrm{C}$ obtained by TDFRS experiment is $D=1.92 \times 10^{7} \mathrm{~cm}^{2} \mathrm{~s}^{-1}$ for water solution and $D=0.92 \times 10^{7} \mathrm{~cm}^{2} \mathrm{~s}^{-1}$ for DMSO solution. These values agree within $12 \%$ with those obtained from DLS measurement as $D=1.71 \times 10^{7}$ $\mathrm{cm}^{2} \mathrm{~s}^{-1}$ for $5.0 \mathrm{gL}^{-1}$ pullulan in water solution and $D=0.81 \times 10^{7} \mathrm{~cm}^{2} \mathrm{~s}^{-1}$ for $5.0 \mathrm{~g} \mathrm{~L}^{-1}$ pullulan in DMSO, respectively. The sign change of $S_{\mathrm{T}}$ of pullulan in water occurs around $40^{\circ} \mathrm{C}$. Although both LS studies of pullulan in water and in DMSO do not show any significant temperature dependence of their thermodynamic properties, the temperature dependence of $S_{\mathrm{T}}$ of pullulan differs 
substantially in water and DMSO.

The thermodynamic properties of pullulan solutions obtained by LS experiments such as $A_{2}$ and $R_{\mathrm{g}} / R_{\mathrm{h}}$ are constant in the investigated temperature range for pullulan in water and in DMSO. A similar behavior has also observed for solutions of PNiPAM in ethanol, where the Soret coefficient also changes its sign, while the solvent quality is good for all investigated temperatures. ${ }^{28}$ These results indicate that the temperature dependence of the Soret coefficient has no direct correlation with the solubility properties determined by LS under homogeneous temperature conditions. This oberservations are in contrast with the results for polyethylenoxide (PEO) in ethanol/water mixtures. For this system a negative Soret coefficient in mixtures with a low water content correlates with bad solvent conditions. While a positive Soret coefficient in pure water corresponds with good solvent conditions. ${ }^{22}$

In general, an increase of solution temperature weakens the formation of hydrogen bondings, thus the negative sign of $S_{\mathrm{T}}$ might be correlated with the solvation of water. This hypothesis is supported by the observations for pullulan in DMSO, which is a polar aprotic solvent not forming hydrogen bonds with the polymer. Here we do not observe a significant temperature dependence of $S_{\mathrm{T}}$. A similar behavior of $S_{\mathrm{T}}$ was reported in another polysaccharide solution, dextran in water, which was the first reported polysaccharide system for which the thermal diffusion has been studied and which shows a sign change with temperatur. ${ }^{24}$ Dextran is mainly composed of $\alpha$ $(1 \rightarrow 6)$-linked glucose with some short $\alpha$-(1 $\rightarrow 3)$-linked glucose branch units. It should be noted that pullulan and dextran that the same smallest constituent, glucose, is the same, but they have different glycosidic bonds to their chains. Both of them are physiologically inactive biopolymers and water soluble. Their fundamental solution properties have been studied well. ${ }^{49,50}$ In Fig. $6 S_{\mathrm{T}}$ of different dextran solutions is plotted in comparison with the results of pullulan in water. Dextran in water ( $\boldsymbol{\square})$ shows a sign change at $45^{\circ} \mathrm{C}$ from negative to positive with increasing temperature. The results are comparable with the results of the pullulan in water (o). Only the slope is slightly steeper compared to the dextran system. The agreement of the temperature dependence of $S_{\mathrm{T}}$ could be originated from chemical contributions of glucose as their basic constituent of pullulan and dextran. For dextran solutions the addition of urea leads to an increase of $S_{\mathrm{T}}$ and the sign change temperature shifts to lower temperatures. It may be assumed that urea destroys the hydrogen bonding ability, thus the addition of urea shifts the Soret coefficient of pullulan towards more positive values which implies that the tendency of pullulan to go to the warm side is enhanced. The effect has the same tendency as an increase of the solution temperature, which also destroys 


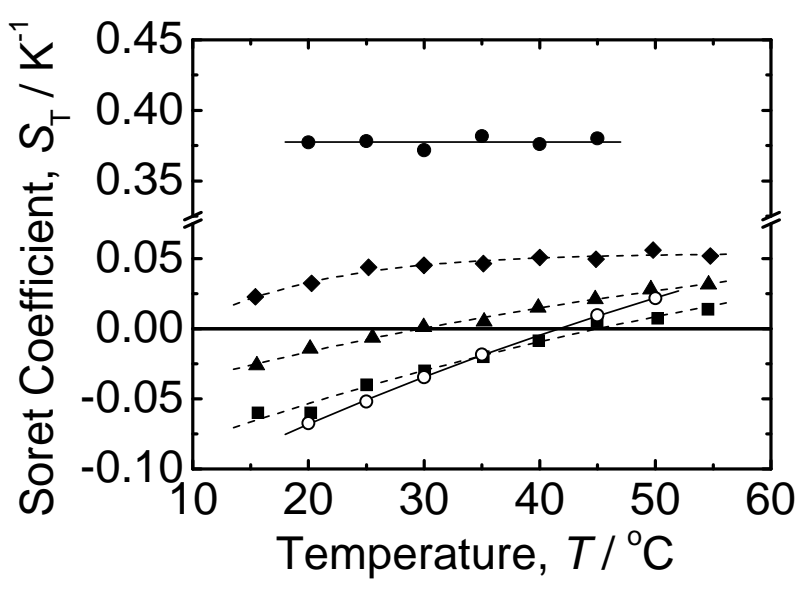

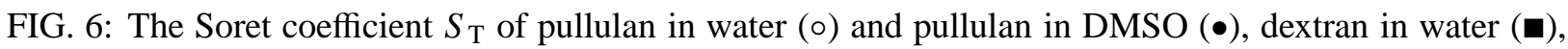
dextran in $2 \mathrm{M}$ urea/water (४) and dextran in $5 \mathrm{M}$ urea/water ( $)$. The results of dextran solutions are obtained by Ref. [24] and the molecular mass of the dextran is reported as $M_{\mathrm{w}}=86.7 \mathrm{~kg} \mathrm{~mol}^{-1}$. The concentration of polysaccharides is $5.0 \mathrm{~g} / \mathrm{L}$ for all solutions. The solid curves are fitted functions according to Eq. 13.

the solvation structure. In other words, a negative sign of $S_{\mathrm{T}}$ implys the formation of a solvation structure via hydrogen bondings. Our preliminary experiment for the aqueous solutions of glucose and maltotriose show that the solutions have a positive sign of $S_{\mathrm{T}}$ and do not show any sign inversion in the same experimental temperature range. These results indicate that the sign change of $S_{\mathrm{T}}$ is not only associated with the chemical contribution of the smallest constituent (? - What do you mean? ) but also with the solution properties of polymeric nature of polysaccharides. The sign change temperatures of the aqueous solutions of pullulan and dextran are at 41.7 and $45.0{ }^{\circ} \mathrm{C}$, which is much higher than the temperature, $T=4^{\circ} \mathrm{C}$, with the largest density of water. For aqueous polysaccharide solutions we do not find a correlation between the thermal expansion coefficient of the solvent and the sign change temperature as suggested by Brenner. ${ }^{31}$

The curves describing the temperature dependence of $S_{\mathrm{T}}$ in Fig. 6 were obtained by a least squares-fit to the empirical equation proposed by Iacopini and Piazza as ${ }^{23}$

$$
S_{\mathrm{T}}(T)=S_{\mathrm{T}}^{\infty}\left[1-\exp \left(\frac{T_{\mathrm{inv}}-T}{T_{0}}\right)\right] .
$$

Here, $S_{\mathrm{T}}^{\infty}$ represents a saturation value of $S_{\mathrm{T}}$ at high temperatures, $T_{\mathrm{inv}}$ is the temperature where $S_{\mathrm{T}}$ changes the sign, and $T_{0}$ indicates to the strength of temperature effects. The obtained parameters of the pullulan and the dextran solutions are tabulated in Table 2. Furthermore, the parameters of 
$S_{\mathrm{T}}^{\infty}, T_{\mathrm{inv}}$, and $T_{0}$ obtained for several polymers, which show a sign change of $S_{\mathrm{T}}$ were collected from literatures and are listed in Table II. As shown in the list, a sign change of $S_{\mathrm{T}}$ with temperature has been found for some proteins, lysozyme, $\beta$-lactoglobuline [BLGA] and poly(L-lysyne), as well as DNA and synthetic polymers, sodium ploy(styrene sulfonate), PEO, and PNiPAM. The solvents, experimental temperature range, and the other experimental conditions are listed together with the references in Table II.

The systems can be divided by the temperature dependent slope of $S_{\mathrm{T}}$ into two groups. In the first group with a positive slope includes $\mathrm{PEO} /$ water/EtOH, PNiPAM/tert-BuOH, $\mathrm{NaPSS} / \mathrm{NaCl} /$ water/, pullulan/water, dextran/water, dextran/urea/water/, lysozyme/salts/buffer solution/, BLGA/NaCl/buffer solution, and DNA/buffer solution. The second group with a negative slope of $S_{\mathrm{T}}$ as a function of temperature consists of PNiPAM/MeOH, PNiPAM/EtOH, PNi$\mathrm{PAM} /$ propanols, and PNiPAM/1-BuOH. Rigolousely speaking, some of them do not show a sign change in the experimentally investigated temperature range $T_{\mathrm{e}}$, but the extrapolated values of sign change temperature could be obtained by a least-square fit to Eq. 13 as listed in Table II. In the case of pullulan/DMSO it is hardly to say, whether there it has a sign inversion temperature, because the system will freeze before it is reached. An overview of all systems is shown in Fig. 7, which shows $S_{\mathrm{T}}$ as function of temperature for the various diluted or semidiluted polymer solutions. For clarity the figure as been splitted in Fig. 7(a) and Fig. 7(b). Here, numbers in the figure refer to the systems as shown in Table II.

Theoretically the temperature dependence of the Soret coefficient is proportional to $1 / T^{2}$ for binary mixture. ${ }^{37,51}$ For aqueous solution a sign change of $S_{\mathrm{T}}$ is related to the thermal expansion of water. ${ }^{44,52}$ Systematic experiments for low molecular mixtures show that the Soret coefficient is proportional to the temperature. ${ }^{33,34} * * *$ All of these earlier investigations of temperature dependence of the Ludwig-Soret effect for non-polymer solutions do not show analogous behavior with the sign change behavior of the aqueous polymers as shown in Fig. 7. For PNiPAM in alcohols the increase of hydrophobic strength such as methanol, ethanol, propanol, and butanol, the magnitude of the Soret coefficient decreased with increasing temperature. PNiPAM in alcohols which obey a weak hydrogen bondings show negative slopes and which indicate the existence a different mechanisms of sign inversion behavior.

If one can focus on biopolymers such as polysaccharide, protein, and DNA, all three polymers have a positive slope with showing sign inversion. The behavior is well described with the em- 


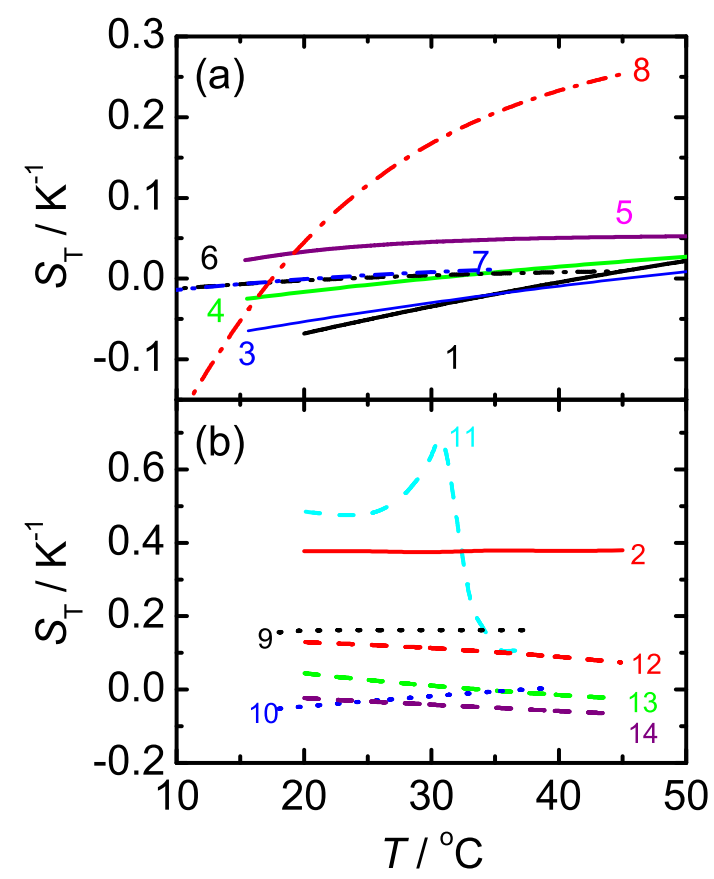

FIG. 7: Soret coefficient of $S_{\mathrm{T}}$ for various solutions as a function of temperature. Curves are drawn using Eq. 13 with the fitting parameters, $S_{\mathrm{T}}^{\infty}, T_{\mathrm{inv}}$, and $T_{0}$ listed in Table II. The numbers refer to the systems which are mentioned in Table II.description of the systems in part (a) and (b) is missing. It became not clear in which way you divided the systems. Another problem is the visibility of the different lines in a black and white copy.

pirical equation proposed by Iacopini and Piazza. ${ }^{23}$ It is interesting to carry out further studies of Ludwig-Soret effect on biological polymers whether there is a correlation between the dissipative phenomena of biopolymers and the function or structures of biopolymers under a temperature gradient. It should be mentioned that the saturation values $S_{\infty}$ and the slope of $S_{\mathrm{T}}$ of the polysaccharides are apparently large in comparison with the other systems of showing the positive slopes such as proteins, NaPSS and PEO as can be seen in Fig. 7, except for DNA. The magnitude of the Soret coefficients of proteins are very small $S_{\mathrm{T}}<0.03 \mathrm{~K}^{-1}$. The studied systems of the aqueous solutions of pullulan and dextran are binary system, though the other systems are ternary or multicomponent systems contain salts and buffer components or alcohol. It indicates that the charges or the additional ingredients to the solution may lead to a modification or disturbance of the local structure of water and which may induce the decrease the strength of magnitude of $S_{\mathrm{T}}$ as well as the slope of $S_{\mathrm{T}}$ against temperature. In fact, the pullulan in DMSO and the dextran in urea/water 
show weaker slope of the Soret coefficient than that of the slope of the binary solutions of pullulan in water and dextran in water. Probably the positive slope of the temperature dependence of the Soret coefficient is associated with the interactions between segments and water, i.e., the solvation is necessary to have a positive slope of $S_{\mathrm{T}}$ and which leads to a sign inversion behavior.

Recently, Stadelmaier and Köhler reported the effect of hydrodynamic interactions with respect to the molecular weight dependence, where the sign inversion of the Soret coefficient is revealed to correlate with the nature of the persistence length of the polymer chain. ${ }^{53}$ This result is comparable with the simulation study of Zhang and Müller-Plathe. ${ }^{53,54}$ These studies are important to understand the mechanism of the sign inversion of the thermal diffusion. Similar studies are desired on biopolymer solutions.

\section{CONCLUSIONS}

The Ludwig-Soret effect of pullulan in water and in DMSO is studied in the temperature range of $20<T_{\mathrm{e}} /{ }^{\circ} \mathrm{C}<50$ for the aqueous solution and $20<T_{\mathrm{e}} /{ }^{\circ} \mathrm{C}<40$ for DMSO solution. The thermally induced sign change of the Soret coefficient was found at the temperature $41.7^{\circ} \mathrm{C}$ for aqueous solutions of pullulan. The temperature above $41.7^{\circ} \mathrm{C}$ pullulan molecules migrate toward the cold side of the fluid $\left(S_{\mathrm{T}}>0\right)$, whereas below $41.7^{\circ} \mathrm{C}$ pullulan molecules migrate toward the hot side $\left(S_{\mathrm{T}}<0\right)$. Contrary, for pullulan in DMSO, where DMSO is a polar aprotic solvent, the sign of $S_{\mathrm{T}}$ is always positive and shows no significant temperature dependence. These results imply that the thermal diffusion of polysaccharide is associated with the strength of hydrogen bonding of polysaccharide solutions. For biopolymers involving polysaccharide, protein, and DNA, which show the positive slope of the Soret coefficient with increase of temperature with a saturation value of $S_{\mathrm{T}}^{\infty}$. Thus, it is expected that the universal behavior of the Soret coefficient as a function of temperature; that is the negative Soret coefficient at lower temperature side is the result of the positive slope against temperature with the saturation value of the Soret coefficient at the higher temperature side. This behavior is well expressed by an empirical function proposed by Iacopini and Piazza. Binary solution of polysaccharide in water has a strong temperature dependence and large saturation values of the Soret coefficient in comparison with protein solutions. It may be arose from the solvation characteristics of the systems. 


\section{ACKNOWLEDGMENTS}

The authors are indebted to Jan Dhont for his kind support and Shin Yagihara and Naoki Shinyashiki for their kind help. The sample (pullulan) donation by Hayashibara co. is gratefully acknowledged. This work is partially supported by Deutsche Forschungsgemeinschaft and by the Ministry of Education, Science, Sports and Culture, Japan (Grant-in-Aid for Scientific Research). 


\section{References}

${ }^{1}$ C. Ludwig. Diffusion zwischen ungleich erwrmten Orten gleich zusammengesetzter Lsungen. Sitz. ber. Akad. Wiss. Wien Math.-naturw. Kl, 20:539, 1856.

2 C. Soret. Sur L'état d'équilibre que prend au point de vue de sa concentration une dissolution saaline primitivement homogène dont deux parties sont portées a des températures différentes. Arch. Geneve, $3: 48,1879$.

3 H.J.V. Tyrell. Diffusion and Heat Flow in Liquids. Butterworth, London, 1961.

4 J.C. Giddings, K.D. Caldwell, and M.N. Myers. Thermal-Diffusion of Polystyrene in 8 Solvents By an Improved Thermal Field-Flow Fractionation Methodology. Macromolecules, 9(1):106-112, 1976.

5 P. M. Shiundu and J. C. Giddings. Influence of bulk and surface composition on the retention of colloidal particles in thermal field-flow fractionation. J. Chromatgr. A, 715:117, 1995.

6 R. Piazza. Thermophoresis: moving particles with thermal gradients. Sft Matter, 4:1740-1744, 2008.

7 S Wiegand. . J. Phys.: Conds. Matter, 16:R357, 2004.

${ }^{8}$ R. Piazza. 'thermal forces': colloids in temperature gradients. JOURNAL OF PHYSICS-CONDENSED MATTER, 16(38):S4195-S4211, 2004.

9 E. Bringuier and A. Bourdon. Colloid transport in nonuniform temperature. Phys. Rev. E, 67(1):011404, 2003. Part 1.

10 J.K.G. Dhont. Thermodiffusion of interacting colloids I: A Statistical Thermodynamic Approach. J. Chem. Phys., 120(3):1632-1641, 2004.

11 A. Parola and R. Piazza. A microscopic approach to thermophoresis in colloidal suspensions. J. Phys.: Condens. Matter, 17:S3639-S3643, 2005.

12 J. K. G. Dhont, S. Wiegand, S. Duhr, and D. Braun. Thermodiffusion of charged colloids: Single-particle diffusion. Langmuir, 23:1674-1683, 2007.

13 A. Würger. Thermophoresis in colloidal suspensions driven by marangoni forces. Phys. Rev. Lett., 98:138301, 2007.

14 J. K. G. Dhont and W. J. Briels. Single-particle thermal diffusion of charged colloids: Double-layer theory in a temperature gradient. Eur Phys J E, 25:61-76, 2008.

15 S. R. deGroot and Mazur P. Non-equilibrium Thermodynamics. Dover, New York, 1984.

16 O. Ecenarro, J.A. Madariaga, J.L. Navarro, C.M. Santamaria, J.A. Carrion, and J.M. Saviron. Ther- 
mogravitational Thermal-Diffusion in Liquid Polymer- Solutions. Macromolecules, 27(18):4968-4971, 1994.

17 W. Köhler, C. Rosenauer, and P. Rossmanith. Holographic Grating Study of Mass and Thermal-Diffusion of Polystyrene Toluene Solutions. Int. J. Thermophys., 16(1):11-21, 1995.

18 G. Z. Zhang, M. E. Briggs, R. W. Gammon, J. V. Sengers, and J. F. Douglas. Thermal and mass diffusion in a semidilute good solvent-polymer solution. J. Chem. Phys., 111(5):2270-2282, 1999.

19 J. Rauch and W. Köhler. Collective and thermal diffusion in dilute, semidilute, and concentrated solutions of polystyrene in toluene. J. Chem. Phys., 119(22):11977-11988, 2003.

20 M. Giglio and A. Vendramini. Soret-Type Motion of Macromolecules in Solution. Phys. Rev. Lett., 38(1):26-30, 1977.

21 B.-J. de Gans, R. Kita, S. Wiegand, and J. Luettmer-Strathmann. To the hot or to the cold? Unusual thermal diffusion in polymer solutions. Phys. Rev. Lett., 91:245501, 2003.

22 B.-J. de Gans, R. Kita, B. Müller, and S. Wiegand. Negative thermodiffusion of polymers and colloids in solvent mixtures. J. Chem. Phys., 118(17):8073-8081, 2003.

23 S. Iacopini and R. Piazza. Thermophoresis in protein solutions. Europhys. Lett., 63(2):247-253, 2003.

${ }^{24}$ R. Sugaya, Wolf. B. A., and R Kita. Thermal diffusion of dextran in aqueous solutions in the absence and the presence of urea. Biomacromolecules, 7(2):435-440, 2006.

25 R. Kita, P. Pavel, and S. Wiegand. Ludwig-soret effect of poly(n-isopropylacrylamide): Temperature dependence study in monohydric alcohols. Macromolecules, 40(5):1638-1642, 2007.

26 J. Luettmer-Strathmann. Two-chamber lattice model for thermodiffusion in polymer solutions. J. Chem. Phys., 119:2892-2902, 2003.

27 R. Kita, S. Wiegand, and J. Luettmer-Strathmann. Sign change of Soret coefficient of poly(ethylene oxide) in water/ethanol mixtures. J. Chem. Phys., 121(8):3874-3885, 2004.

${ }^{28}$ R. Kita, G. Kircher, and S. Wiegand. Thermally induced sign change of soret coefficient for dilute and semidilute solutions of poly(n-isopropylacrylamide) in ethanol. J. Chem. Phys., 121(18):9140-9146, 2004.

29 S. Duhr and D. Braun. Why molecules move along a temperature gradient. Proc. Natl. Acad. Sci. U.S.A.,, 103(52):19678-19682, 2006.

30 S. Wiegand, H. Ning, and R. Kita. 'universal concentration dependenc of the soret coefficient in aqueous systems. JOURNAL OF NON-EQUILIBRIUM THERMODYNAMICS, 32(3):193-201, 2007.

$31 \mathrm{H}$. Brenner. Elementary kinetical model of thermal diffusion in liquid and gases. Phys. Rev. E, 
74:036306, 2006.

${ }^{32} \mathrm{H}$. Ning and S. Wiegand. Experimental investigation of the Soret effect in acetone/water and water/dimethylsulfoxide mixtures. J. Chem. Phys., 125:221102, 2006.

33 P. Polyakov, J. Luettmer-Strathmann, and S. Wiegand. Study of the thermal diffusion behavior of alkane/benzene mixtures by thermal diffusion forced rayleigh scattering experiments and lattice model calculations. J. Phys. Chem. B, 110(51):26215-26224, 2006.

34 G. Wittko and W. Köhler. On the temperature dependence of thermal diffusion of liquid mixtures. Europhys. Lett., 78(4):46007, 2007.

35 J. S. Ham. Kinetic theory of thermal diffusion in dilute polymer solutions. J. Appl. Phys., 31:1853-1858, 1960.

36 T. N. Khazanovich. On the theory of thermal diffusion in dilute polymer solutions. J. Polym. Sci. C, 16:2463-2468, 1967.

$37 \mathrm{H}$. Li and L. Wu. On the relationship between thermal diffusion and molecular interaction energy in binary mixtures. J. Phys. Chem. B, 108:13821-13826, 2004.

38 S. L. Brimhall, M. N. Myers, K. D. Calldwell, and J. C. Giddings. Study of temperature dependence of thermal diffusion in polystyrene/ethylbenzen by thermal field-flow fractionation. J. Polym. Sci. C: Polym. Phys., 23:2443-2456, 1985.

39 E. P. C. Mes, W. Th. Kok, and R. Tijssen. Prediction of polymer thermal diffusion coefficients from polymer-solvent interaction parameters: Comparison with thermal field flow fractionation and thermal diffusion forced rayleigh scattering experiments. International Journal of Polymer Anal. Charact., 8:133-153, 2003.

${ }^{40}$ F. C. Whitmore. Experimental study of thermal diffusion in dilute solutions of high polymers. J. Appl. Phys., 31:1858-1864, 1960.

41 J. Luettmer-Strathmann. Thermodiffusion in the critical region. In W. Köhler and S. Wiegand, editors, Thermal nonequilibrium phenomena in fluid mixtures, Lecture Notes in Physics, pages 24-37, Berlin, 2002. Springer.

42 S. Wiegand. Thermal diffusion in liquid mixtures and polymer solutions. J.Phys.:Condens. Matter, 16:R357-R379, 2004.

${ }^{43}$ R. Kita and S. Wiegand. Soret coefficient of poly(n-isopropylacrylamide)/water in the vicinity of coilglobule transition temperature. Macromolecules, 38(11):4554-4556, 2005.

44 S. Iacopini, R. Rusconi, and R. Piazza. The "macromolecular tourist": Universal temperature depen- 
dence of thermal diffusion in aqueous colloidal suspensions. Eur. Phys. J., E 19:59-67, 2006.

45 W. Köhler and R. Schäfer. Polymer analysis by thermal-diffusion forced rayleigh scattering. In New Developments in Polymer Analytics II, volume 151 of Advances in Polymer Science, pages 1-59. Springer, Berlin, 2000.

46 S. Wiegand, H. Ning, and H. Kriegs. Thermal Diffusion Forced Rayleigh Scattering Setup Optimized for Aqueous Mixtures. J. Phys. Chem. B, 111:14169-14174, 2007.

47 A. Becker, W. Köhler, and B. Müller. A Scanning Michelson Interferometer For the Measurement of the Concentration and Temperature Derivative of the Refractive- Index of Liquids. Ber. Bunsen-Ges. Phys. Chem. Chem. Phys., 99(4):600-608, 1995.

48 T. Kato, K. Tsunehisa, and T. Akira. Static and dynamic solution properties of pullulan in a dilute solution. Macromolecules, 17(9):1727-1730, 1984.

49 E. Nordmeier. Static and dynamic light-scattering solution behavior of pullulan and dextran in comparison. J. Phys. Chem., 97(21):5770-5785, 1993.

50 A. Rolland-Sabat é, M. G. Mendez-Montealvo, P. Colonna, and V. Planchot. Online determination of structural properties and observation of deviations from power law behavior. Biomacromolecules, 9:1719-1730, 2008.

51 L. Hang and W. Laosheng. On the relationship between thermal diffusion and molecular interaction energy in binary mixtures. J. Phys. Chem. B, 108:13821-13826, 2004.

52 H. Brener. Elementary kinematical model of thermal diffusion in liquids and gases. Phys. Rev. E, 74:036306, 2006.

53 D. Stadelmaier and W. Köhler. From Small Molecules to High Polymers: Investigation of the Crossover of Thermal Diffusion in Dilute Polystyrene Solutions. Macromolecules, 41(16):6205-6209, 2008.

54 M. Zhang and F. Müller-Plathe. The soret effect in dilute polymer solutions: influence of chain length, chain stiffness and solvent quality. J. Chem. Phys., 125:124903, 2006. 
TABLE I: Molecular parameters of pullulan in water and in DMSO determined by static and dynamic light scattering.

\begin{tabular}{|c|c|c|c|c|c|c|c|c|c|c|}
\hline Solvents & $\begin{array}{c}T \\
{ }^{\circ} \mathrm{C} \\
\end{array}$ & $\begin{array}{c}10^{-5} M_{\mathrm{W}} \\
/ \mathrm{g} \mathrm{mol}^{-1} \\
\end{array}$ & $\begin{array}{c}10^{4} A_{2} \\
/ \mathrm{cm}^{3} \mathrm{~mol} \mathrm{~g}^{-2} \\
\end{array}$ & $\begin{array}{l}\left\langle R_{\mathrm{g}}\right\rangle \\
/ \mathrm{nm} \\
\end{array}$ & $\begin{array}{l}10^{7}\left\langle D_{0}\right\rangle \\
/ \mathrm{cm}^{2} \mathrm{~s}^{-1}\end{array}$ & $\begin{array}{l}\left\langle R_{\mathrm{h}}\right\rangle \\
/ \mathrm{nm}\end{array}$ & $\left\langle R_{\mathrm{g}}\right\rangle /\left\langle R_{\mathrm{h}}\right\rangle$ & $\begin{array}{c}k_{\mathrm{d}} \\
/ \mathrm{cm}^{3} \mathrm{~g}^{-1} \\
\end{array}$ & $\begin{array}{c}k_{\mathrm{f}} \\
/ \mathrm{cm}^{3} \mathrm{~g}^{-1} \\
\end{array}$ & $\mu_{2} / \bar{\Gamma}^{2}$ \\
\hline \multirow[t]{8}{*}{ Water } & 20.3 & 4.67 & 1.75 & 32.0 & 1.21 & 17.9 & 1.79 & 30.5 & 28.3 & 0.30 \\
\hline & 25.0 & 4.40 & 1.43 & 33.0 & 1.36 & 18.2 & 1.82 & 46.7 & 41.7 & 0.31 \\
\hline & 29.7 & 4.29 & 1.07 & 32.9 & 1.52 & 18.3 & 1.80 & 48.2 & 42.7 & 0.36 \\
\hline & 35.0 & 4.37 & 1.51 & 33.6 & 1.69 & 18.7 & 1.79 & 60.5 & 51.6 & 0.32 \\
\hline & 40.0 & 4.15 & 1.62 & 33.5 & 1.91 & 18.5 & 1.81 & 59.7 & 52.2 & 0.33 \\
\hline & 45.0 & 4.23 & 1.76 & 32.8 & 2.16 & 18.1 & 1.81 & 51.1 & 45.8 & 0.25 \\
\hline & 50.1 & 4.72 & 1.96 & 32.6 & 2.41 & 17.9 & 1.82 & 47.8 & 41.4 & 0.24 \\
\hline & 55.0 & 4.52 & 1.71 & 32.5 & 2.64 & 17.9 & 1.82 & 42.8 & 38.2 & 0.39 \\
\hline \multirow[t]{5}{*}{ DMSO } & 20.0 & 3.36 & 4.24 & 34.0 & 0.46 & 21.4 & 1.59 & 96.7 & 45.5 & 0.36 \\
\hline & 25.0 & 3.45 & 4.19 & 36.2 & 0.51 & 21.8 & 1.66 & 96.1 & 44.8 & 0.32 \\
\hline & 30.0 & 3.57 & 4.17 & 33.4 & 0.59 & 20.7 & 1.62 & 88.8 & 44.2 & 0.34 \\
\hline & 35.0 & 3.65 & 4.04 & 34.7 & 0.63 & 21.7 & 1.60 & 102.3 & 47.2 & 0.29 \\
\hline & 40.0 & 3.49 & 4.03 & 34.0 & 0.71 & 21.5 & 1.58 & 96.7 & 46.3 & 0.34 \\
\hline
\end{tabular}


TABLE II: The values of $S_{\mathrm{T}}^{\infty}, T_{\mathrm{inv}}$, and $T_{0}$ obtained from Eq. 13 for various synthetic polymer and biopolymer solutions. I would kick out those systems, which are not discussed in the figure. The table ned to be reduced so that it fits on a page of the journal. Maybe you can leave out the experimental temperature range and the molecular weight. For the solvent you could use a minipage command. I put an example in the solvent column of lysozyme.

\begin{tabular}{|c|c|c|c|c|c|c|c|c|c|}
\hline Solute & $\begin{array}{c}M_{\mathrm{W}} \\
/ \mathrm{kg} \mathrm{mol}^{-1}\end{array}$ & Solvent & $\begin{array}{l}\text { Concentration } \\
\qquad / \mathrm{g} \mathrm{mol}^{-1}\end{array}$ & $\begin{array}{l}S_{\mathrm{T}}^{\infty} \\
/ \mathrm{K}^{-1}\end{array}$ & $\begin{array}{c}T_{\mathrm{inv}} \\
/ \mathrm{K}\end{array}$ & $\begin{array}{l}T_{0} \\
/ \mathrm{K}\end{array}$ & $\begin{array}{l}\text { Experimental temperature } \\
\text { range } /{ }^{\circ} \mathrm{C}\end{array}$ & $\begin{array}{l}\text { Numbers refer to } \\
\text { the curve in Fig. } 7\end{array}$ & Ref. \\
\hline \multirow[t]{2}{*}{ pullulan } & \multirow[t]{2}{*}{440} & water & \multirow[t]{2}{*}{5.0} & 0.23 & 314.8 & 84.9 & $20<T_{\mathrm{e}}<50$ & 1 & \multirow[t]{2}{*}{ This worl } \\
\hline & & DMSO & & - & - & - & $20<T_{\mathrm{e}}<45$ & 2 & \\
\hline \multirow[t]{5}{*}{ dextran } & \multirow[t]{5}{*}{86.7} & water & 1.0 & 0.04 & 314.2 & 21.2 & $15<T_{\mathrm{e}}<55$ & - & \multirow[t]{5}{*}[18]{} \\
\hline & & water & 5.0 & 0.13 & 318.2 & 71.4 & $15<T_{\mathrm{e}}<55$ & 3 & \\
\hline & & water & 10.0 & 0.08 & 318.6 & 53.1 & $15<T_{\mathrm{e}}<55$ & - & \\
\hline & & $2 \mathrm{M}$ urea/water & 5 & 0.10 & 302.9 & 63.5 & $15<T_{\mathrm{e}}<55$ & 4 & \\
\hline & & $5 \mathrm{M}$ urea/water & 5 & 0.05 & 282.5 & 11.1 & $15<T_{\mathrm{e}}<55$ & 5 & \\
\hline \multirow[t]{5}{*}{ lysozyme } & \multirow[t]{5}{*}{14} & $\begin{array}{c}7.5 \mathrm{mM} \mathrm{NaCl} / \text { water } \\
(\mathrm{pH}=4.65)\end{array}$ & 7 & 0.0203 & 288.7 & 26 & $5<T_{\mathrm{e}}<45$ & - & \multirow[t]{5}{*}{ [17] } \\
\hline & & $20 \mathrm{mM} \mathrm{NaCl} /$ water & 7 & 0.0212 & 293.8 & 28 & $5<T_{\mathrm{e}}<45$ & - & \\
\hline & & $\begin{array}{c}(\mathrm{pH}=4.65) \\
100 \mathrm{mM} \mathrm{NaCl} / \text { water }\end{array}$ & & & & & & & \\
\hline & & $\begin{array}{c}(\mathrm{pH}=4.65) \\
400 \mathrm{mM} \mathrm{NaCl} / \text { water }\end{array}$ & 7 & 0.0190 & 298.0 & 26 & $5<T_{\mathrm{e}}<45$ & - & \\
\hline & & $(\mathrm{pH}=4.65)$ & 7 & 0.0143 & 296.0 & 21 & $5<T_{\mathrm{e}}<45$ & 6 & \\
\hline \multirow[t]{9}{*}{ lysozyme $* 1$} & \multirow[t]{9}{*}{14} & $100 \mathrm{mM} \mathrm{NaCl} /$ water $(\mathrm{pH}=3)$ & 10 & 0.0128 & 299.2 & 22 & $6<T_{\mathrm{e}}<35$ & - & \multirow[t]{9}{*}{ [46] } \\
\hline & & $100 \mathrm{mM} \mathrm{NaCl} /$ water $(\mathrm{pH}=4.55)$ & 10 & 0.0129 & 297.3 & 22 & $6<T_{\mathrm{e}}<35$ & - & \\
\hline & & $100 \mathrm{mM} \mathrm{NaCl} /$ water $(\mathrm{pH}=7.1)$ & 10 & 0.0125 & 296.0 & 18 & $6<T_{\mathrm{e}}<35$ & - & \\
\hline & & $100 \mathrm{mM} \mathrm{NaCl} /$ water $(\mathrm{pH}=9.3)$ & 10 & 0.0182 & 297.9 & 25 & $6<T_{\mathrm{e}}<35$ & - & \\
\hline & & $100 \mathrm{mM} \mathrm{NaCl} /$ water $(\mathrm{pH}=4.65)$ & 10 & 0.0110 & 297.6 & 20 & $6<T_{\mathrm{e}}<35$ & - & \\
\hline & & $100 \mathrm{mM} \mathrm{KCl} /$ water $(\mathrm{pH}=4.65)$ & 10 & 0.0105 & 294.7 & 17 & $6<T_{\mathrm{e}}<35$ & - & \\
\hline & & $100 \mathrm{mM} \mathrm{LiCl} /$ water $(\mathrm{pH}=4.65)$ & 10 & 0.0159 & 291.9 & 20 & $6<T_{\mathrm{e}}<37$ & - & \\
\hline & & $100 \mathrm{mM} \mathrm{KBr} /$ water $(\mathrm{pH}=4.65)$ & 10 & 0.0102 & 294.4 & 19 & $6<T_{\mathrm{e}}<37$ & - & \\
\hline & & $100 \mathrm{mM} \mathrm{KNO}_{3} /$ water $(\mathrm{pH}=4.65)$ & 10 & 0.0105 & 296.0 & 19 & $6<T_{\mathrm{e}}<37$ & - & \\
\hline$\beta$-lactoglobuline & 36 & $50 \mathrm{mM} \mathrm{NaCl} /$ water $(\mathrm{pH}=7.0)$ & 13 & 0.0275 & 293.9 & 26 & $10<T_{\mathrm{e}}<35$ & 7 & [35] \\
\hline poly(1-lysyne) & 50 & $100 \mathrm{mM} \mathrm{NaCl} /$ water $(\mathrm{pH}=7.0)$ & 5.4 & 0.0340 & 294.6 & 26 & $10<T_{\mathrm{e}}<34$ & - & [35] \\
\hline pMD31 plasmid DNA & - & $7.5 \mathrm{mM}$ phosphate buffer $(\mathrm{pH}=7.0)$ & 0.6 & 0.3701 & 288.3 & 26 & $10<T_{\mathrm{e}}<45$ & 8 & [35] \\
\hline \multirow[t]{3}{*}{ NaPSS } & 15.2 & $100 \mathrm{mM} \mathrm{NaCl} /$ water & 2 & 0.0470 & 276.2 & 20 & $6<T_{\mathrm{e}}<36$ & - & \multirow[t]{3}{*}{ [35] } \\
\hline & 32.9 & $100 \mathrm{mM} \mathrm{NaCl} /$ water & 2 & 0.0550 & 276.9 & 19 & $6<T_{\mathrm{e}}<37$ & - & \\
\hline & 74 & $100 \mathrm{mM} \mathrm{NaCl} /$ water & 2 & 0.0720 & 277.0 & 22 & $6<T_{\mathrm{e}}<36$ & - & \\
\hline \multirow[t]{5}{*}{ PEO } & \multirow[t]{5}{*}{265} & $\mathrm{EtOH} /$ water $(w=1.0)$ & 5 & 0.1623 & 286.2 & 2 & $18<T_{\mathrm{e}}<38$ & 9 & \multirow[t]{5}{*}{ [21] } \\
\hline & & $\mathrm{EtOH} /$ water $(w=0.90)$ & 5 & 0.1166 & 275.8 & 20 & $18<T_{\mathrm{e}}<38$ & - & \\
\hline & & $\mathrm{EtOH} /$ water $(w=0.85)$ & 5 & 0.0625 & 291.4 & 17 & $18<T_{\mathrm{e}}<38$ & - & \\
\hline & & $\mathrm{EtOH} /$ water $(w=0.82)$ & 5 & 0.0889 & 301.3 & 35 & $18<T_{\mathrm{e}}<38$ & - & \\
\hline & & $\mathrm{EtOH} /$ water $(w=0.80)$ & 5 & 0.1813 & 310.4 & 76 & $18<T_{\mathrm{e}}<40$ & 10 & \\
\hline PNiPAM & 3000 & water & 1.0 & - & - & - & $20<T_{\mathrm{e}}<38$ & 11 & [34] \\
\hline \multirow[t]{4}{*}{ PNiPAM } & \multirow[t]{4}{*}{3000} & $\mathrm{EtOH}$ & 0.2 & -0.4966 & 307.6 & 69 & $14<T_{\mathrm{e}}<40$ & - & \multirow[t]{4}{*}{ [22] } \\
\hline & & $\mathrm{EtOH}$ & 1.0 & -0.3066 & 305.4 & 50 & $14<T_{\mathrm{e}}<40$ & - & \\
\hline & & $\mathrm{EtOH}$ & 2.0 & -0.6004 & 305.6 & 82 & $14<T_{\mathrm{e}}<40$ & - & \\
\hline & & $\mathrm{EtOH}$ & 5.0 & -0.2005 & 305.6 & 43 & $14<T_{\mathrm{e}}<40$ & - & \\
\hline \multirow[t]{6}{*}{ PNiPAM } & 1200 & $\mathrm{MeOH}$ & 10.0 & 0.1642 & 333.6 & -26 & $20<T_{\mathrm{e}}<45$ & 12 & [19] \\
\hline & & $\mathrm{EtOH}$ & 10.0 & -0.0882 & 307.0 & 34 & $20<T_{\mathrm{e}}<45$ & 13 & \\
\hline & & 1-PrOH & 10.0 & -0.1096 & 285.8 & 40 & $20<T_{\mathrm{e}}<45$ & - & \\
\hline & & 2-PrOH & 10.0 & 0.2139 & 259.6 & -211 & $20<T_{\mathrm{e}}<45$ & - & \\
\hline & & 1-BuOH & 10.0 & -0.7646 & 284.9 & 345 & $20<T_{\mathrm{e}}<45$ & 14 & \\
\hline & & tert-BuOH & 10.0 & 0.0092 & 445.8 & 55 & $20<T_{\mathrm{e}}<45$ & - & \\
\hline
\end{tabular}

\footnotetext{
$* 1$ The fitting parameters are obtained by a scanning and digitizing figure of photocopied articles, thus the values might have a slight deviation from the original data of respective authors.
} 